\title{
Recent patents in CRISPR technology
}

Patent number

US $9,234,213$

ngineered CRISPR-Cas9 systems for genomic modification in mammalian cells. Design and testing of a polynucleotide encoding the Streptococcus pyogenes protein in which the nucleotide sequence has been optimized for expression in mammalian cells. Also, all-in-one systems for RNA-guided genome engineering in mammalian cells, including human cells.

US 9,228,208 Compositions and methods for modifying a genomic locus of interest in a eukaryotic cell, a mammalian cell, a human cell, or a nonhuman mammalian cell using a large targeting vector (LTVEC) comprising various endogenous or exogenous nucleic acid sequences; compositions and methods for generating a genetically modified nonhuman animal comprising one or more targeted genetic modifications in their germline. Further methods combine the use of the LTVEC with a CRISPR-Cas system.

US 9,149,049 A genetic cluster of strains of Streptococcus thermophilus, wherein the milk fermented with said strains is highly viscous and weakly ropy. Also, a genetic cluster of strains of $S$. thermophilus, wherein the genome of said strains comprises a CRISPR locus having the nucleotide sequence of Seq ID No. 1.

US 9,102,936 A method of processing a target RNA, comprising contacting the products of an RNA-ligase-mediated ligation reaction with a CAS6 protein, wherein: (i) the RNA-ligase-mediated ligation reaction comprises: a target RNA, an RNA ligase, and first and second adaptors that can ligate together to produce an adaptor dimer that contains a CRISPR stem loop; and (ii) the CAS6 protein recognizes the CRISPR stem loop; thereby preventing the adaptor dimer from being reverse transcribed.

US 8,999,641 Engineering and optimization of systems, methods, and compositions for manipulation of sequences and/or activities of target sequences. Provided are vectors and vector systems, some of which encode one or more components of a CRISPR complex, as well as methods for the design and use of such vectors with additional functional domains.

US 8,945,839 Systems, methods, and compositions for altering the expression of target gene sequences and related gene products. Provided are vectors and vector systems, some of which encode one or more components of a CRISPR complex, as well as methods for the design and use of such vectors. Also provided are methods of directing CRISPR complex formation in eukaryotic cells and methods for utilizing the CRISPR-Cas system.

US 8,932,814 Systems, methods, and compositions for manipulation of sequences and/or activities of target sequences. Provided are vectors and vector systems, some of which encode one or more components of a CRISPR complex, as well as methods for the design and use of such vectors. Also provided are methods of directing CRISPR complex formation in eukaryotic cells and methods for selecting specific cells by introducing precise mutations utilizing the CRISPR-Cas system.

US 8,906,616 Engineering of systems, methods, and optimized guide compositions for sequence manipulation. Systems, methods, and compositions for manipulation of sequences and/or activities of target sequences.

US 8,895,308 Engineering and optimization of systems, methods, and compositions for manipulation of sequences and/or activities of target sequences. Provided are compositions and methods related to components of a CRISPR complex particularly comprising a Cas ortholog enzyme.

US 8,871,445 CRISPR-Cas component systems, methods, and compositions for sequence manipulation. Systems, methods, and compositions for manipulation of sequences and/or activities of target sequences. Provided are vectors and vector systems, some of which encode one or more components of a CRISPR complex, as well as methods for the design and use of such vectors. Also provided are methods of directing CRISPR complex formation in eukaryotic cells and methods for selecting specific cells by introducing precise mutations utilizing the CRISPR-Cas system.

Assignee

System B
CA, USA)

Inventor

Date

$1 / 12 / 2016$

Regeneron Pharmaceuticals

(Tarrytown, NY, USA)
Frendewey D,

Auerbach W,

Lai K-MV,

Valenzuela DM,

Yancopoulos GD
DuPont Nutrition Biosciences

(Copenhagen)

Agilent Technologies (Santa Clara, CA, USA)
Manoury E,

Horvath $\mathrm{P}$,

Fremaux C,

Fourcassie $P$

Zeiner G,

Bruhn L

$10 / 6 / 2015$

$1 / 5 / 2016$

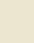

